\title{
A Novel Wide-Band Reflection-Based System for Measuring Abdominal Fat in Humans
}

\author{
Siamak Sarjoghian, Yasir Alfadhl, Xiaodong Chen \\ School of Electronic Engineering and Computer Science \\ Queen Mary University of London \\ London E1 4NS, UK \\ s.sarjoghian@qmul.ac.uk
}

\begin{abstract}
A Wide-Band (WB) radio frequency (RF) system has been thoroughly developed in order to measure the Subcutaneous Fat (SF) thickness in the abdominal region of humans. Recent conducted research has shown that the SF thickness is related to the thickness of internal fat called Visceral Fat (VF), which has important health implications, and therefore cannot be measured accurately by non-invasive techniques. Thus, this proposed noninvasive RF system offers the possibility of measuring the SF thickness and then estimating the VF thickness. The investigation details work carried out to optimise the penetration depth and the range resolution. These considerations resulted in a Gaussian pulse with a $1.45 \mathrm{GHz}$ centre frequency and $462 \mathrm{MHz}$ bandwidth being selected for the system. This has been implemented using an accurately designed compact antenna based on double-ridged horn (DRH) inside a high-permittivity material, which has been designed using the CST Microwave Studio (MWS) software. The employed abdominal model consisted of three layers: skin; fat, and muscle. The WB pulse has been directed at the model, and the reflections recorded for several SF thicknesses, including an infinitely thick layer, which provided the reference system pulse to compare the other cases with. This successfully demonstrated the principles of the system operation, with SF thickness of 10 to $30 \mathrm{~mm}$ being accurately measured, based on amplitude variations and also time shifting, in the proposed RF system.
\end{abstract}

Keywords_Double-ridged horn; Subcutaneous fat; Visceral fat; wide-band.

\section{INTRODUCTION}

Obesity or increasing weight in humans as they reach middle age is one of the main concerns of the health watch specialists, as the proportion of fat in the body is related to poor health [1]; increasing the risk of a range of health dilemmas such as metabolic disorders, hyperlipidemia, hypertension, diabetes mellitus, and cardiovascular disease [2]. Most of these fat cells will accumulate and be stored in the upper body especially the abdomen, waist and thighs. Abdominal fat is more relevant to health care as these fats are closer to important organs such as liver, spleen, gallbladder, kidneys, and other internal organs. These fats can act like a gland or endocrine organ that produce hormones and other substances like leptin which release after meal to dampens appetite, adiponectin that influence the response of cells to insulin, and substances like cytokines. These hormones can boost the risk of cardiovascular disease by promulgating insulin resistance and triggering low level inflammation, a risk factor for heart disease and other chronic conditions. While obesity can also play a critical role in a person's self-esteem and confidence [3].

Research and clinical experiments over accumulating of the abdominal fat and its correlation to the health risk make researchers and health watchers keen to be able to measure amount of the fat in each layer. There are few techniques which are developed in order to measure the quantity of fat such as: Magnetic Resonance Imaging (MRI) [6]; Computed Tomography (CT) [7]; Multi-Frequency Body Composition Analyser (MC-180MA) [8]; and other techniques [9-10].

The proposed RF system uses WB technology which involves high bandwidth radio-waves (typically $\sim 500 \mathrm{MHz}$ ), and was originally aimed at the short-range wireless communication channels. This technology requires a low power level and can be used for short-range or indoor applications. It utilises a large portion of radio spectrum that can improve the speed, resolution, and reduce interference of data transfer compared with other current technology available on the market.

This study tries to highlight the fact that the measurement of the SF is possible using the proposed WB technique which has improvements over other known techniques such as eliminates ionizing effect, improving resolution, reducing cost. Also, the research is motivated by the previous research conducted on the dielectrically scaled antenna and matching the source pulse with respect to target pulse for biomedical sensors [11]. The aim of this research is to measure the thicknesses of SF using the WB signals, and therefore VF in the abdominal region.

In the abdominal region, the SF layer is between the skin and the muscle. For normal average age humans, previous research has estimated thickness of each of the layers as approximately $1.5-2.5 \mathrm{~mm}$ of skin, $16 \mathrm{~mm}$ of SF, and $20-30 \mathrm{~mm}$ of muscle [5]. The permittivity of each of these layers is given in table 1 . The information is used to determine the preliminary model, which has the following layer thicknesses: $2 \mathrm{~mm}$ of skin, 10$30 \mathrm{~mm}$ of SF, and infinite muscle. In practice, because of the penetration depth and the permittivity of the muscle, there are no reflections expected from anything behind the muscle, and therefore the infinite muscle thickness is just a convenient boundary condition for the proposed system model.

\section{System Modelling AND Methodology}

This section discusses the system design issues. There are some important potential factors that are taken into account to achieve a functioning RF system for the intended objectives. 
Pulse shape: Each pulse has its own shape that can be used for different purposes based on the application such as data rate and resolution.

Centre frequency: The pulse centre frequency is important, as it can determine the penetration depth of pulse into a medium. Bandwidth of pulse can determine the system resolution and how easy it is to implement.

\section{A. Pulse Type}

After some research two types of pulses were considered for the system. The step time (ST) pulse and the Gaussian pulse. However, after further research and simulation, the ST pulse is found to have several disadvantages, such as the energy not decaying very rapidly, as the time signal never stops [12]. Hence, the pulse is then selected to be used in the system. The Gaussian pulse is named after the Gauss function due to the similarity of their mathematical definitions, as shown in equation (1) where ' $s$ ' is defined as the standard deviation:

$$
\stackrel{\text { yields }}{\longrightarrow} G(x)=\frac{1}{\sqrt{2 \pi s^{2}}} e^{-x^{2} / 2 x s^{2}}
$$

Gaussian pulses are normally used in ultra-wideband (UWB) systems, and have a very wide bandwidth. However, in this system, this type of pulse can be used with a reduced bandwidth, as it is still gives acceptable results. The pulse is initially produced at low frequency, and then is modulated with a high frequency sine-wave to shift to high frequency.

\section{B. Penetration Depth}

The propagation constant of the each model layer can be defined as $(\gamma=\alpha+j \beta)$, where $\alpha$ is the attenuation constant, $\beta$ is the phase constant, and $\delta$ is the skin depth constant which can be defined by equations (2)-(4), respectively [13]:

$$
\begin{aligned}
& \alpha=\omega \sqrt{\mu_{0} \varepsilon_{0} \varepsilon_{r}}\left[\frac{1}{2}\left(\sqrt{1+\left(\frac{\sigma}{\omega \varepsilon_{0} \varepsilon_{r}}\right)^{2}}-1\right)\right]^{\frac{1}{2}}(N P / m) \\
& \beta=\omega \sqrt{\mu_{0} \varepsilon_{0} \varepsilon_{r}}\left[\frac{1}{2}\left(\sqrt{1+\left(\frac{\sigma}{\omega \varepsilon_{0} \varepsilon_{r}}\right)^{2}}+1\right)\right]^{\frac{1}{2}}(\mathrm{rad} / \mathrm{m}) \\
& \delta=\frac{1}{\alpha}=\frac{1}{\omega \sqrt{\mu_{0} \varepsilon_{0} \varepsilon_{r}}\left[\frac{1}{2}\left(\sqrt{1+\left(\frac{\sigma}{\omega \varepsilon_{0} \varepsilon_{r}}\right)^{2}}+1\right)\right]^{\frac{1}{2}}}(\mathrm{~m})
\end{aligned}
$$

where $\sigma$ is the conductivity; $\omega=2 \pi f$ is the angular frequency, $\varepsilon_{0}$ is the permittivity of free space, and $\mu_{0}$ is the permeability of free space.

In considering the frequency dependent penetration depth of each tissue type, a pulse centre frequency of $\sim 1.45 \mathrm{GHz}$ is considered as the optimum value. Table 1 lists the penetration depth, derived from these equations, at this frequency. These values are also used throughout the paper for characterisation.

\section{Dielectric Properties}

The tissue types are essentially treated as dielectrics and as such the material properties are characterise by the relative permittivity $(\varepsilon)$ and conductivity $(\sigma)$. The complex relative permittivity is defined as equation (5):

$$
\varepsilon=\varepsilon^{\prime}-j \varepsilon^{\prime \prime}
$$

where, $\varepsilon^{\prime}$ is the real part of the relative permittivity, and $\varepsilon^{\prime \prime}$ is the imaginary part or the loss factor, and is given by (6) as:

$$
\varepsilon^{\prime \prime}=\frac{\sigma}{\varepsilon_{0} \omega}
$$

The reaction of any material to the electric field comprises of a physical shift of charge and also the kinetics of the shift. This varies with frequency and gives rise to the material properties. This reaction can be modelled either in terms of a resonance or a relaxation. The Debye model is employed in order to characterise the conductivity in terms of a relaxation time $(\tau)$. The first and second order of Debye equations are given in equations (7) and (8); and are used to analyse the dielectric properties of biological tissue in various frequencies [13].

$$
\begin{gathered}
\varepsilon(\omega)=\varepsilon_{\infty}+\frac{\varepsilon_{s}-\varepsilon_{\infty}}{1+j \omega \tau} \\
\varepsilon(\omega)=\varepsilon_{\infty}+\frac{\varepsilon_{s 1}-\varepsilon_{\infty}}{1+j \omega \tau_{1}}+\frac{\varepsilon_{s 2}-\varepsilon_{\infty}}{1+j \omega \tau_{2}}
\end{gathered}
$$

where, $\varepsilon(\omega)$ is the complex permittivity; $\varepsilon_{s}$ is relative static permittivity at low frequencies; and $\varepsilon_{\infty}$ is the relative optical permittivity at high frequencies.

Table 1. The dielectric parameters and penetration depth of three types of humans' tissues at $1.45 \mathrm{GHz}$ [14].

\begin{tabular}{|c|c|c|c|c|}
\hline $\begin{array}{c}\text { Tissue } \\
\text { Name }\end{array}$ & $\begin{array}{c}\text { Frequency } \\
{[\mathbf{G H z}]}\end{array}$ & $\begin{array}{c}\text { Conductivity } \\
{[\mathbf{S} / \mathbf{m}]}\end{array}$ & $\begin{array}{c}\text { Relative } \\
\text { Permittivity }\end{array}$ & $\begin{array}{c}\text { Penetration } \\
\text { Depth [mm] }\end{array}$ \\
\hline Dry Skin & 1.45 & 1.05 & 39.5 & 32 \\
\hline Wet Skin & 1.45 & 1.06 & 44.5 & 33 \\
\hline Fat & 1.45 & 0.07 & 5.4 & 186 \\
\hline Muscle & 1.45 & 1.16 & 54 & 34 \\
\hline
\end{tabular}

\section{System Overview}

System is benefiting from enormous numbers of features in which some has been specified and discussed transitory previously. In this part of the paper some of these features that have more impact on the system will be pointed out to express the novelty of the system. The first and important element that the system is using during its operation is the WB pulse that found as a potential candidate based on the features such as illuminating ionising effect, low power, and therefore low cost as well as being safe for the biomedical systems. The centre frequency of below $1.5 \mathrm{GHz}$ and bandwidth of $\sim 500 \mathrm{MHz}$ characterised as a significant centre frequency and bandwidth based on the satisfactory penetration depth and range resolution provide for the RF system. The DRH antenna has been picked based on its superlative aspects such as directivity and gain and has been scaled down by using the technique of integrating the antenna in the ceramic with permittivity close to the skin. The technique will eliminate the huge expect reflection from the interaction of the skin with the free space. However, in practice 
there is a need for the gel with the same permittivity in order to illuminate the remaining mismatch. Finally, the pulse has been transmitted through the proposed model and the reflected pulse is recorded and is subtracted from the reflected pulse which is result of the interaction of the skin and SF layer that achieves in the simulation by simply placing the infinite layer of the SF in the RF model. This removes all the unwanted reflected pulse from the received pulse which left the system with the intended reflected pulse that result of interaction of the SF layer with the muscle layer. The results are then compared with the reference produced graphs result to estimate the thickness of SF layer.

\section{SySTEM SimULATIONS}

\section{A. System Design and Simulation Results}

The DRH antenna is designed in the CST-MWS software with the background material of a high permitivity $\left(\varepsilon_{r}=41\right)$ which makes the design compact with dimensions as: $L=29.845 ; W$ $=32.435 ; H=26.48$; and angle of $18.08^{\circ}$. The antenna has 462 $\mathrm{MHz}$ bandwidth within $-10 \mathrm{~dB}$ point operating between 1.19 $\mathrm{GHz}-1.65 \mathrm{GHz}$. The centre frequency of the design has its best performance at $1.45 \mathrm{GHz}$ that goes to $-28 \mathrm{~dB}$ point, along with directivity gain of around $9.76 \mathrm{~dB}$ at its centre frequency, which all are acceptable for the proposed RF system.

In order to specify the optimum location for the antenna with respect to the model, the according calculations are performed for the far-field region, as in equation (9):

$$
\begin{gathered}
\stackrel{\text { yields }}{\longrightarrow} \lambda=c / f \sqrt{\varepsilon_{r}}=32.3(\mathrm{~mm}) ; \\
d_{f}=2 \times D^{2} /_{\lambda}=\frac{2 \times 32^{2}}{32.3}=63.4(\mathrm{~mm}) .
\end{gathered}
$$

where, $\lambda$ is the wavelength; $c$ is is the speed of light; $f$ is the centre frequency; $\varepsilon_{r}$ is the permittivity of the environment of the antenna; $d_{f}$ is the distance of tissue from the antenna; and $D$ is the maximum dimension of the aperture of antenna. The modelled tissue has been placed $60 \mathrm{~mm}$ apart from the antenna feeding that has been found as an effective region with respect to the far-field region and the penetration depth (i.e., fig. 1).

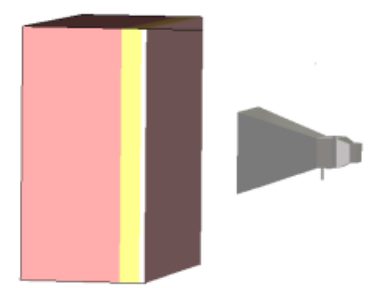

Fig. 1. The tissue model is placed $60 \mathrm{~mm}$ apart from the DRH antenna.

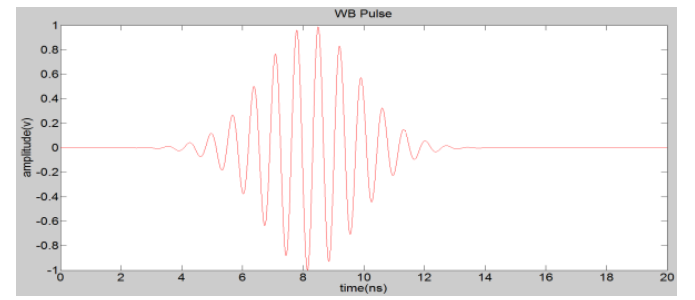

Fig. 2. The transmitted Gaussian pulse with: $f_{L}=1.19 \mathrm{GHz} ; f_{H}=1.65 \mathrm{GHz}$.
As fig. 2 presents the Gaussian pulse is generated using the CST-MWS by selecting the lower and higher RF frequencies (i.e., 1.19-1.65 GHz) in the time-solver.

The proposed RF approach is to first transmit the pulse to the model using the DRH antenna, and then to record the received pulse, with respect to the knowledge of the received pulse (i.e. the combination of all the reflected ones formed by interaction of one layer to the other). The next step is then to extract the reflected pulse that formed from interaction of the SF layer with the muscle layer from the overall received pulse. This is being implemented in the simulation by changing the model structure to have infinite SF layer, where the transmitted pulse will be absorbed within the SF region. Hence, the reflected RF pulse contains all reflection up to the SF layer as the skin layer thickness and the distance of the antenna from the model more or less stay the same, and finally this pulse is subtracted from the overall received pulse. The subtraction procedure outcome is in fact the only reflected pulse from the interaction of the SF layer with muscle layer, and is generated using the MATLAB. This process has been repeated for five model cases where the SF thickness iterates 5 times from $10-30 \mathrm{~mm}$, in which each case the value of $5 \mathrm{~mm}$ SF is added to the previous case and then results have been transferred back to the MATLAB to be subtracted from the infinite SF layer case individually (fig. 3). Figs. 4 (a) and (b) present the estimated table values for the amplitude, and the time, respectively. The amplitude drop and also the time delay in all the cases where the SF layer has been differed from $10 \mathrm{~mm}$ to $30 \mathrm{~mm}$ by iteration thickness of $5 \mathrm{~mm}$ at the highest picks within the expected time frame have been recorded and then generated based on fig. 3 (b). The graphs have been generated using the Excel software for both tables, in order to be able to generate the equations that can predict the unknown points in both cases (i.e. amplitude and time); as well as the graph based on changes in the thickness of the SF layer (which is used as a reference point). The results of a number of points that are obtained based on the generated equations mentioned previously, are then simulated and shown the approximate agreement which can prove this RF approach.

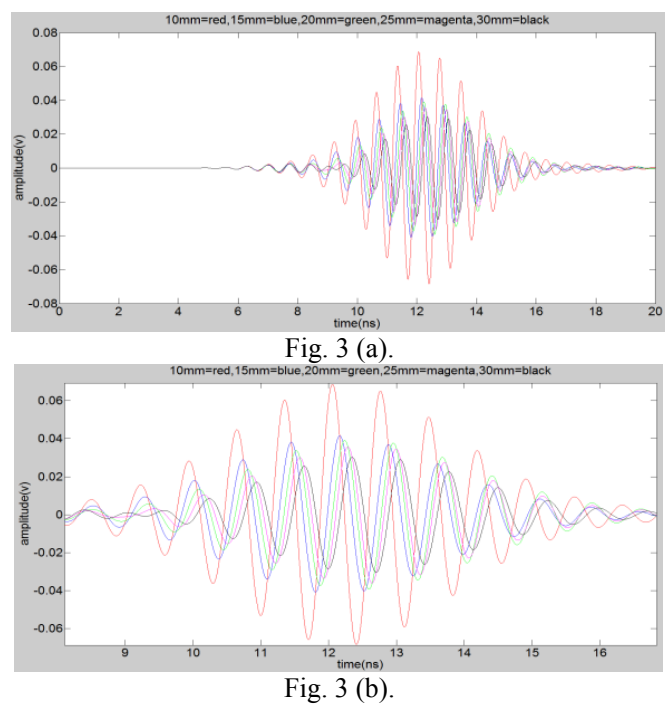

Fig. 3. The reflected pulses, in the five scenarios that fat layer thickness is increased from $10 \mathrm{~mm}$ (red) to $30 \mathrm{~mm}$ (black) with $5 \mathrm{~mm}$ iterations. 

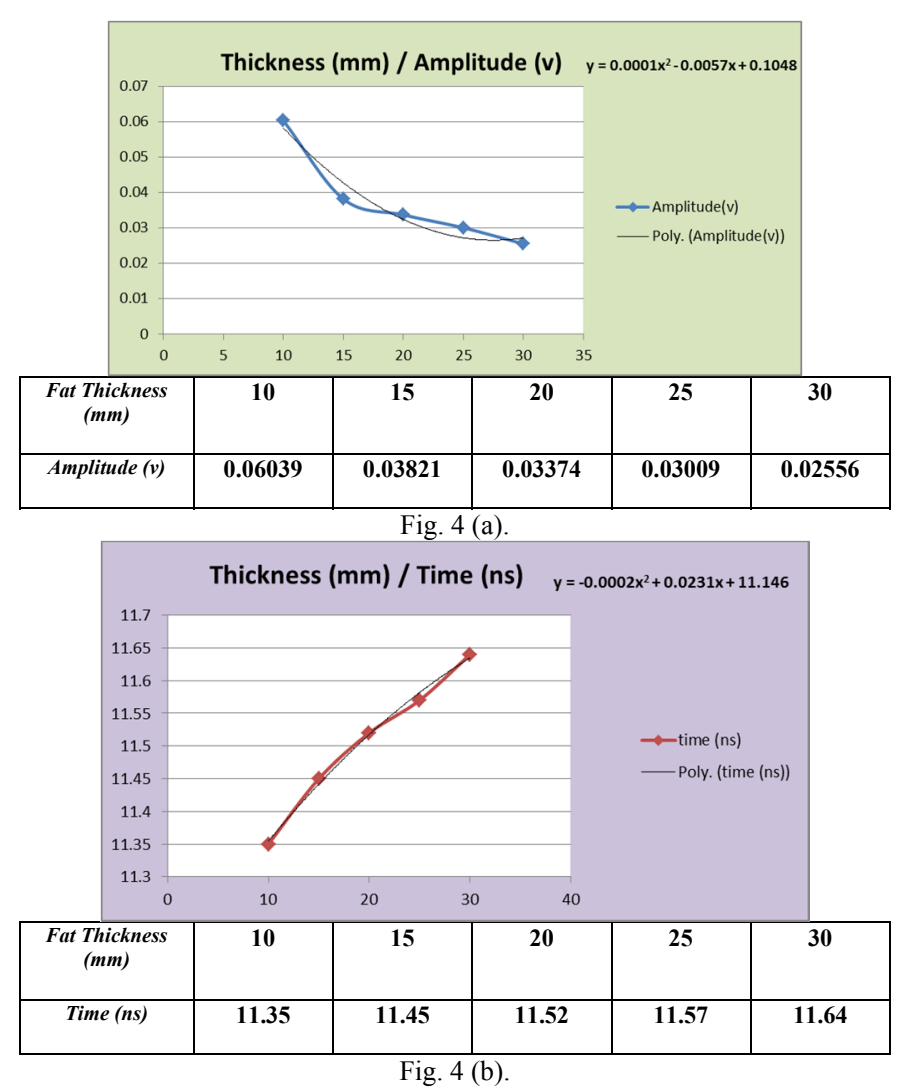

Fig. 4. The graphs and equations generated based on the reflected pulses in all the scenarios in terms of the amplitude [fig.4 (a)], and the time [fig.4 (b)] change with respect to different thicknesses.

\section{DISCUSSIONS}

The developed methodologies and the presented simulations are in well agreement according to the following issues:

The selected Gaussian pulse with a centre frequency of 1.45 $\mathrm{GHz}$ that refers to the penetration depth of the pulse and the bandwidth of $462 \mathrm{MHz}$ that refers to the system resolution have significant performance with respect to the equations as well as simulation. By moving centre frequency and therefore bandwidth to lower or higher frequencies one of the important factors (penetration depth or resolution) may affect the overall RF system performance.

The proposed method of emerging the antenna inside the high permittivity material is used in the system to not only scaling down the RF antenna size; more over to illuminate the huge reflection result of interaction of the free space with the skin layer.

The technique of the reflected-based system in time-domain has been used previously based on the overall reflected pulse; however, in this proposed technique it is benefiting from the extracting specific SF layer pulse from the overall reflected pulses in order to classify the amplitude change and also time shifting, which can increase the resolution and hence rise of the accuracy.
This RF technique employs the known data retrieved from the mediums such as skin thickness and their behaviour to the electromagnetic (EM) field to extract the unknown data, such as SF thickness, from the overlapped data.

\section{CONCLUSION}

The time-domain results demonstrate that the measurement of the SF layer within the range thickness of $10-30 \mathrm{~mm}$ is possible based on the proposed WB system; by extracting the data from the RF reflected pulses using subtraction technique of common layers reflection from the overall reflected pulse. The WB technique proves that this technique is in fact potentially useful for the future RF-based biomedical systems and devices in order to measure and detect the SF layer along with other tissue layers. The presented simulation results will also be validated against the known patient data, as part of the future work.

\section{REFERENCES}

[1] A. H. Kissebah et al., "Relation of body fat distribution to metabolic complications of obesity," J. Clin. Endocrinol. Metab., vol. 54, no. 2, 254-260, Feb. 1982.

[2] B. Larsson et al., "Abdominal adipose tissue distribution, obesity, and risk of cardiovascular disease and death: 13 year follow up of participants in the study of men born in 1913," Br. Med. J. (Clin. Res. Ed.), vol. 288, pp. 1401-1404, May 1984.

[3] Harvard Medical School, "Abdominal fat and what to do about it," Harvard Health Publications [Online], Oct. 2015.

[4] B. L. Wajchenberg, "Subcutaneous and Visceral Adipose Tissue: Their Relation to the Metabolic Syndrome" Endocr. Rev., vol. 21, no. 6, pp. 697-738, Dec. 2000.

[5] G. Rankin, "Abdominal muscle size and symmetry in normal subject," Institute of Complex Neuro-disability, Royal Hospital for Neuro-Disability, London, UK, 2006.

[6] E. G. Kamel et al., "Measurement of abdominal fat by magnetic resonance imaging, dual-energy X-ray absorptiometry and anthropometry in non-obese men and women," Int. J. Obes. Relat. Metab. Disord., vol. 23, no. 7, pp. 686-692, Jul. 1999.

[7] T. Yoshizumi et al., "Abdominal fat: standardized technique for measurement at CT," Radiology, vol. 211, no. 1, 283-286, 1999.

[8] Tanita Health Equipments, "Multi-Frequency Body Composition Analyzer (MC-180MA)," Tanita Corporation, 2005.

[9] M. Tengvall et al., "Body composition in the elderly reference values and bioelectrical impedance spectroscopy to predict total body skeletal muscle mass," Clin Nutr., vol. 28, no. 1, pp. 52-58, Feb. 2009.

[10] B. Yang et al., "Body Fat Detection Using Radio Frequency," School of EECS, Queen Mary University of London, Jun. 2009.

[11] U. Schwarz et al., "Design and application of dielectrically scaled double-ridged horn antennas for biomedical UWB radar applications," Ilmenau University of Technology, Sep. 2009.

[12] CST MICROWAVE STUDIO, EM Simulation Software 2015 version [Online]. Available: CST2013: Signals in Time Domain Simulations.

[13] G. Kang and O. P. Gandh, "Effect of dielectric properties on the peak 1- and 10-g SAR for $802.11 \mathrm{a} / \mathrm{b} / \mathrm{g}$ frequencies 2.45 and 5.15 to $5.85 \mathrm{GHz}$," IEEE Trans. on Electromagnetic Compatibility, vol. 46, no. 2, pp. 268-274, May 2004.

[14] D. Andreuccetti and R. Fossi, "Dielectric properties of human tissues definitions, parametric model, and computer codes," Report N.TR/ICEMM/13.00, IFAC-CNR, Firenze (I), Sep. 2000.

[15] Y. Alfadhl, "Numerical evaluations on the interaction of electromagnetic fields with animals and biological tissues," School of EECS, Queen Mary University of London, 2005. 